\title{
MNase Sequencing
}

National Cancer Institute

\section{Source}

National Cancer Institute. MNase Sequencing. NCI Thesaurus. Code C106056.

A molecular genetic technique where genome-wide sequencing is performed on

chromosomal DNA that is resistant to treatment with micrococcal nuclease. This technique identifies nucleosomal DNA sequences. 